\title{
Blow-up criterion of smooth solutions for magneto-micropolar fluid equations with partial viscosity
}

\author{
Yu-Zhu Wang ${ }^{*}$, Yifang Li and Yin-Xia Wang
}

\author{
* Correspondence: yuzhu108@163. \\ com \\ School of Mathematics and \\ Information Sciences, North China \\ University of Water Resources and \\ Electric Power, Zhengzhou 450011, \\ China
}

\section{Abstract}

In this paper, we investigate the Cauchy problem for the incompressible magnetomicropolar fluid equations with partial viscosity in $\mathbb{R}^{n}(n=2,3)$. We obtain a BealeKato-Majda type blow-up criterion of smooth solutions.

MSC (2010): 76D03; $35 \mathrm{Q} 35$.

Keywords: magneto-micropolar fluid equations, smooth solutions; blow-up criterion

\section{Introduction}

The incompressible magneto-micropolar fluid equations in $\mathbb{R}^{n}(n=2,3)$ takes the following form

$$
\left\{\begin{array}{l}
\partial_{t} u-(\mu+\chi) \Delta u+u \cdot \nabla u-b \cdot \nabla b+\nabla\left(p+\frac{1}{2}|b|^{2}\right)-\chi \nabla \times v=0, \\
\partial_{t} v-\gamma \Delta v-\kappa \nabla \operatorname{div} v+2 \chi v+u \cdot \nabla v-\chi \nabla \times u=0, \\
\partial_{t} b-v \Delta b+u \cdot \nabla b-b \cdot \nabla u=0, \\
\nabla \cdot u=0, \quad \nabla \cdot b=0,
\end{array}\right.
$$

where $u(t, x), v(t, x), b(t, x)$ and $p(t, x)$ denote the velocity of the fluid, the microrotational velocity, magnetic field and hydrostatic pressure, respectively. $\mu, \chi, \gamma, \kappa$ and $v$ are constants associated with properties of the material: $\mu$ is the kinematic viscosity, $\chi$ is the vortex viscosity, $\gamma$ and $\kappa$ are spin viscosities, and $\frac{1}{v}$ is the magnetic Reynold. The incompressible magneto-micropolar fluid equations (1.1) has been studied extensively (see [1-8]). Rojas-Medar [5] established the local in time existence and uniqueness of strong solutions by the spectral Galerkin method. Global existence of strong solution for small initial data was obtained in [4]. Rojas-Medar and Boldrini [6] proved the existence of weak solutions by the Galerkin method, and in 2D case, also proved the uniqueness of the weak solutions. Wang et al. [2] obtained a Beale-Kato-Majda type blow-up criterion for smooth solution $(u, v, b)$ to the magneto-micropolar fluid equations with partial viscosity that relies on the vorticity of velocity $\nabla \times u$ only (see also [8]). For regularity results, refer to Yuan [7] and Gala [1].

If $b=0,(1.1)$ reduces to micropolar fluid equations. The micropolar fluid equations was first proposed by Eringen [9]. It is a type of fluids which exhibits the micro-rotational effects and micro-rotational inertia, and can be viewed as a non-Newtonian

(c) 2011 Wang et al; licensee Springer. This is an Open Access article distributed under the terms of the Creative Commons Attribution License (http://creativecommons.org/licenses/by/2.0), which permits unrestricted use, distribution, and reproduction in any medium, provided the original work is properly cited. 
fluid. Physically, micropolar fluid may represent fluids that consisting of rigid, randomly oriented (or spherical particles) suspended in a viscous medium, where the deformation of fluid particles is ignored. It can describe many phenomena appeared in a large number of complex fluids such as the suspensions, animal blood, liquid crystals which cannot be characterized appropriately by the Navier-Stokes equations, and that it is important to the scientists working with the hydrodynamic-fluid problems and phenomena. For more background, we refer to [10] and references therein. The existences of weak and strong solutions for micropolar fluid equations were treated by Galdi and Rionero [11] and Yamaguchi [12], respectively. The global regularity issue has been thoroughly investigated for the 3D micropolar fluid equations and many important regularity criteria have been established (see [13-19]). The convergence of weak solutions of the micropolar fluids in bounded domains of $\mathbb{R}^{n}$ was investigated (see [20]). When the viscosities tend to zero, in the limit, a fluid governed by an Eulerlike system was found.

If both $v=0$ and $\chi=0$, then Equations 1.1 reduces to be the magneto-hydrodynamic (MHD) equations. The local well-posedness of the Cauchy problem for the incompressible MHD equations in the usual Sobolev spaces $H^{s}\left(\mathbb{R}^{3}\right)$ is established in [21] for any given initial data that belongs to $H^{s}\left(\mathbb{R}^{3}\right), s \geq 3$. But whether this unique local solution can exist globally is a challenge open problem in the mathematical fluid mechanics. There are numerous important progresses on the fundamental issue of the regularity for the weak solution to (1.1), (1.2) (see [22-34]). In this paper, we consider the magneto-micropolar fluid equations (1.1) with partial viscosity, i.e., $\mu=\chi=0$. Without loss of generality, we take $\gamma=\kappa=v=1$. The corresponding magneto-micropolar fluid equations thus reads

$$
\left\{\begin{array}{l}
\partial_{t} u+u \cdot \nabla u-b \cdot \nabla b+\nabla\left(p+\frac{1}{2}|b|^{2}\right)=0 \\
\partial_{t} v-\Delta v-\nabla \operatorname{div} v+u \cdot \nabla v=0 \\
\partial_{t} b-\Delta b+u \cdot \nabla b-b \cdot \nabla u=0 \\
\nabla \cdot u=0, \quad \nabla \cdot b=0
\end{array}\right.
$$

We obtain a blow-up criterion of smooth solutions to (1.2), which improves our previous result (see [2]).

In the absence of global well-posedness, the development of blow-up/non-blow-up theory is of major importance for both theoretical and practical purposes. For incompressible Euler and Navier-Stokes equations, the well-known Beale-Kato-Majda's criterion [35] says that any solution $u$ is smooth up to time $T$ under the assumption that $\int_{0}^{T}\|\nabla \times u(t)\|_{L^{\infty}} d t<\infty$. Beale-Kato-Majda's criterion is slightly improved by Kozono et al. [36] under the assumption $\int_{0}^{T}\|\nabla \times u(t)\|_{B M O} d t<\infty$. In this paper, we obtain a Beale-Kato-Majda type blow-up criterion of smooth solutions to Cauchy problem for the magneto-micropolar fluid equations (1.2).

Now, we state our results as follows.

Theorem 1.1 Assume that $u_{0}, v_{0}, b_{0} \in H^{m}\left(\mathbb{R}^{n}\right)(n=2,3), m \geq 3$ with $\nabla \cdot u_{0}=0, \nabla$. $b_{0}=0$. Let $(u, v, b)$ be a smooth solution to Equations 1.2 with initial data $u(0, x)=u_{0}$ $(x), v(0, x)=v_{0}(x), b(0, x)=b_{0}(x)$ for $0 \leq t<T$. If $u$ satisfies 


$$
\int_{0}^{T}\|\nabla \times u(t)\|_{\dot{B}_{\infty, \infty}^{0}} d t<\infty
$$

then the solution $(u, v, b)$ can be extended beyond $t=T$.

We have the following corollary immediately.

Corollary 1.1 Assume that $u_{0}, v_{0}, b_{0} \in H^{m}\left(\mathbb{R}^{n}\right)(n=2,3), m \geq 3$ with $\nabla \cdot u_{0}=0, \nabla$. $b_{0}=0$. Let $(u, v, b)$ be a smooth solution to Equations 1.2 with initial data $u(0, x)=u_{0}$ $(x), v(0, x)=v_{0}(x), b(0, x)=b_{0}(x)$ for $0 \leq t<T$. Suppose that $T$ is the maximal existence time, then

$$
\int_{0}^{T}\|\nabla \times u(t)\|_{\dot{B}_{\infty, \infty}^{0}} d t=\infty
$$

The plan of the paper is arranged as follows. We first state some preliminary on functional settings and some important inequalities in Section 2 and then prove the blow-up criterion of smooth solutions to the magneto-micropolar fluid equations (1.2) in Section 3.

\section{Preliminaries}

Let $\mathcal{S}\left(\mathbb{R}^{n}\right)$ be the Schwartz class of rapidly decreasing functions. Given $f \in \mathcal{S}\left(\mathbb{R}^{n}\right)$, its Fourier transform $\mathcal{F} f=\hat{f}$ is defined by

$$
\hat{f}(\xi)=\int_{\mathbb{R}^{n}} e^{-i x \cdot \xi} f(x) d x
$$

and for any given $g \in \mathcal{S}\left(\mathbb{R}^{n}\right)$, its inverse Fourier transform $\mathcal{F}^{-1} g=\breve{g}$ is defined by

$$
\breve{g}(x)=\int_{\mathbb{R}^{n}} e^{i x \cdot \xi} g(\xi) d \xi .
$$

In what follows, we recall the Littlewood-Paley decomposition. Choose a non-negative radial functions $\phi \in \mathcal{S}\left(\mathbb{R}^{n}\right)$, supported in $\mathcal{C}=\left\{\xi \in \mathbb{R}^{n}: \frac{3}{4} \leq|\xi| \leq \frac{8}{3}\right\}$ such that

$$
\sum_{k=-\infty}^{\infty} \phi\left(2^{-k} \xi\right)=1, \quad \forall \xi \in \mathbb{R}^{n} \backslash\{0\} .
$$

The frequency localization operator is defined by

$$
\Delta_{k} f=\int_{\mathbb{R}^{n}} \breve{\phi}(y) f\left(x-2^{-k} y\right) d y .
$$

Next, we recall the definition of homogeneous function spaces (see [37]). For $(p, q) \in$ $[1, \infty]^{2}$ and $s \in \mathbb{R}$, the homogeneous Besov space $\dot{B}_{p, q}^{s}$ is defined as the set of $f$ up to polynomials such that

$$
\|f\|_{\dot{B}_{p, q}^{s}} \triangleq\left\|2^{k s}\right\| \Delta_{k} f\left\|_{L^{p}}\right\|_{\left.l q^{q} \mathbb{Z}\right)}<\infty
$$

In what follows, we shall make continuous use of Bernstein inequalities, which comes from [38].

Lemma 2.1 For any $s \in \mathbb{N}, 1 \leq p \leq q \leq \infty$ and $f \in L^{p}\left(\mathbb{R}^{n}\right)$, then the following inequalities 


$$
c 2^{k m}\left\|\Delta_{k} f\right\|_{L^{p}} \leq\left\|\nabla^{m} \Delta_{k} f\right\|_{L^{p}} \leq C 2^{k m}\left\|\Delta_{k} f\right\|_{L^{p}}
$$

and

$$
\left\|\Delta_{k} f\right\|_{L^{q}} \leq C 2^{n\left(\frac{1}{p}-\frac{1}{q}\right) k}\left\|\Delta_{k} f\right\|_{L^{p}}
$$

hold, where $c$ and $C$ are positive constants independent of $f$ and $k$.

The following inequality is well-known Gagliardo-Nirenberg inequality.

Lemma 2.2 Let $j, m$ be any integers satisfying $0 \leq j<m$, and let $1 \leq q, r \leq \infty$, and $p \in \mathbb{R}, \frac{j}{m} \leq \theta \leq 1$ such that

$$
\frac{1}{p}-\frac{j}{n}=\theta\left(\frac{1}{r}-\frac{m}{n}\right)+(1-\theta) \frac{1}{q} .
$$

Then for all $f \in L^{q}\left(\mathbb{R}^{n}\right) \cap W^{m, r}\left(\mathbb{R}^{n}\right)$, there is a positive constant $C$ depending only on $n, m, j, q, r, \theta$ such that the following inequality holds:

$$
\left\|\nabla^{j} f\right\|_{L^{p}} \leq C\|f\|_{L^{q}}^{1-\theta}\left\|\nabla^{m} f\right\|_{L^{r}}^{\theta}
$$

with the following exception: if $1<r<1$ and $m-j-\frac{n}{r}$ is a nonnegative integer, then (2.3) holds only for a satisfying $\frac{j}{m} \leq \theta<1$.

The following lemma comes from [39].

Lemma 2.3 Assume that $1<p<\infty$. For $f, g \in W^{m, p}$, and $1<q_{1}, q_{2} \leq \infty, 1<r_{1}, r_{2}<1$, we have

$$
\left\|\nabla^{\alpha}(f g)-f \nabla^{\alpha} g\right\|_{L^{p}} \leq C\left(\|\nabla f\|_{L^{q_{1}}}\left\|\nabla^{\alpha-1} g\right\|_{L^{r_{1}}}+\|g\|_{L^{q_{2}}}\left\|\nabla^{\alpha} f\right\|_{L^{r_{2}}}\right),
$$

where $1 \leq \alpha \leq m$ and $\frac{1}{p}=\frac{1}{q_{1}}+\frac{1}{r_{1}}=\frac{1}{q_{2}}+\frac{1}{r_{2}}$.

Lemma 2.4 There exists a uniform positive constant $C$, such that

$$
\|\nabla f\|_{L^{\infty}} \leq C\left(1+\|f\|_{L^{2}}\|\nabla \times f\|_{\dot{B}_{\infty, \infty}^{0}} \ln \left(e+\|f\|_{H^{3}}\right)\right) .
$$

holds for all vectors $f \in H^{3}\left(\mathbb{R}^{n}\right)(n=2,3)$ with $\nabla \cdot f=0$.

Proof. The proof can be founded in [36]. For the convenience of the readers, the proof will be also sketched here. It follows from Littlewood-Paley composition that

$$
\nabla f=\sum_{k=-\infty}^{0} \Delta_{k} \nabla f+\sum_{k=1}^{A} \Delta_{k} \nabla f+\sum_{k=A+1}^{\infty} \Delta_{k} \nabla f .
$$

Using (2.1), ( 2.2) and (2.6), we obtain

$$
\begin{aligned}
\|\nabla f\|_{L^{\infty}} \leq & \sum_{k=-\infty}^{0}\left\|\Delta_{k} \nabla f\right\|_{L^{\infty}}+\left\|\sum_{k=1}^{A} \Delta_{k} \nabla f\right\|_{L^{\infty}}+\sum_{k=A+1}^{\infty}\left\|\Delta_{k} \nabla f\right\|_{L^{\infty}} \\
\leq & C \sum_{k=-\infty}^{0} 2^{\left(1+\frac{n}{2}\right) k}\left\|\Delta_{k} f\right\|_{L^{2}}+A \max _{1 \leq k \leq A}\left\|\Delta_{k} \nabla f\right\|_{L^{\infty}+} \\
& \sum_{k=A+1}^{\infty} 2^{-\left(2-\frac{n}{2}\right) k}\left\|\Delta_{k} \nabla^{3} f\right\|_{L^{2}} \\
\leq & C\left(\|f\|_{L^{2}}+A\|\nabla f\|_{\dot{B}_{\infty, \infty}^{0}}+2^{-\left(2-\frac{n}{2}\right) A}\left\|\nabla^{3} f\right\|_{L^{2}}\right) .
\end{aligned}
$$


Taking

$$
A=\left[\frac{1}{\left(2-\frac{n}{2}\right) \ln 2} \ln \left(e+\|f\|_{H^{3}}\right)\right]+1 .
$$

It follows from (2.7), (2.8) and Calderon-Zygmand theory that (2.5) holds. Thus, we have completed the proof of lemma.

In order to prove Theorem 1.1, we need the following interpolation inequalities in two and three space dimensions.

Lemma 2.5 In three space dimensions, the following inequalities

$$
\left\{\begin{array}{l}
\|\nabla f\|_{L^{2}} \leq C\|f\|_{L^{2}}^{\frac{2}{3}}\left\|\nabla^{3} f\right\|_{L^{2}}^{\frac{1}{3}} . \\
\|f\|_{L^{\infty}} \leq C\|f\|_{L^{2}}^{\frac{1}{4}}\left\|\nabla^{2} f\right\|_{L^{2}}^{\frac{3}{4}} . \\
\|f\|_{L^{4}} \leq C\|f\|_{L^{2}}^{\frac{3}{4}}\left\|\nabla^{3} f\right\|_{L^{2}}^{\frac{1}{4}}
\end{array}\right.
$$

hold, and in two space dimensions, the following inequalities

$$
\left\{\begin{array}{l}
\|\nabla f\|_{L^{2}} \leq C\|f\|_{L^{2}}^{\frac{2}{3}}\left\|\nabla^{3} f\right\|_{L^{2}}^{\frac{1}{3}} . \\
\|f\|_{L^{\infty}} \leq C\|f\|_{L^{2}}^{\frac{1}{2}}\left\|\nabla^{2} f\right\|_{L^{2}}^{\frac{1}{2}} . \\
\|f\|_{L^{4}} \leq C\|f\|_{L^{2}}^{\frac{5}{6}}\left\|\nabla^{3} f\right\|_{L^{2}}^{\frac{1}{6}}
\end{array}\right.
$$

hold.

Proof. (2.9) and (2.10) are of course well known. In fact, we can obtain them by Sobolev embedding and the scaling techniques. In what follows, we only prove the last inequality in (2.9) and (2.10). Sobolev embedding implies that $H^{3}\left(\mathbb{R}^{n}\right), \otimes L^{4}\left(\mathbb{R}^{n}\right)$ for $n=$ 2 , 3. Consequently, we get

$$
\|f\|_{L^{4}} \leq C\left(\|f\|_{L^{2}}+\left\|\nabla^{3} f\right\|_{L^{2}}\right) .
$$

For any given $0 \neq f \in H^{3}\left(\mathbb{R}^{n}\right)$ and $\delta>0$, let

$$
f_{\delta}(x)=f(\delta x) .
$$

By (2.11) and (2.12), we obtain

$$
\left\|f_{\delta}\right\|_{L^{4}} \leq C\left(\left\|f_{\delta}\right\|_{L^{2}}+\left\|\nabla^{3} f_{\delta}\right\|_{L^{2}}\right),
$$

which is equivalent to

$$
\|f\|_{L^{4}} \leq C\left(\delta^{-\frac{n}{4}}\|f\|_{L^{2}}+\delta^{3-\frac{n}{4}}\left\|\nabla^{3} f\right\|_{L^{2}}\right) .
$$

Taking $\delta=\|f\|_{L^{2}}^{\frac{1}{3}}\left\|\nabla^{3} f\right\|_{L^{2}}^{-\frac{1}{3}}$ and $n=3$ and $n=2$, respectively. From (2.14), we immediately get the last inequality in (2.9) and (2.10). Thus, we have completed the proof of Lemma 2.5. ㅁ

\section{Proof of main results}

Proof of Theorem 1.1. Adding the inner product of $u$ with the first equation of (1.2), of $v$ with the second equation of (1.2) and of $b$ the third equation of (1.2), then using integration by parts, we get 


$$
\frac{1}{2} \frac{d}{d t}\left(\|u(t)\|_{L^{2}}^{2}+\|v(t)\|_{L^{2}}^{2}+\|b(t)\|_{L^{2}}^{2}\right)+\|\nabla v(t)\|_{L^{2}}^{2}+\|\operatorname{div} v(t)\|_{L^{2}}^{2}+\|\nabla b(t)\|_{L^{2}}^{2}=0
$$

where we have used $\nabla \cdot \cdot u=0$ and $\nabla \cdot b=0$.

Integrating with respect to $t$, we have

$$
\begin{aligned}
& \|u(t)\|_{L^{2}}^{2}+\|v(t)\|_{L^{2}}^{2}+\|b(t)\|_{L^{2}}^{2}+2 \int_{0}^{t}\|\nabla v(\tau)\|_{L^{2}}^{2} d \tau+2 \int_{0}^{t}\|\operatorname{div} v(\tau)\|_{L^{2}}^{2} d \tau+ \\
& 2 \int_{0}^{t}\|\nabla b(\tau)\|_{L^{2}}^{2} d \tau=\left\|u_{0}\right\|_{L^{2}}^{2}+\left\|v_{0}\right\|_{L^{2}}^{2}+\left\|b_{0}\right\|_{L^{2}}^{2} .
\end{aligned}
$$

Applying $\nabla$ to (1.2) and taking the $L^{2}$ inner product of the resulting equation with $(\nabla u, \nabla v, \nabla b)$, with help of integration by parts, we have

$$
\begin{aligned}
& \frac{1}{2} \frac{d}{d t}\left(\|\nabla u(t)\|_{L^{2}}^{2}+\|\nabla v(t)\|_{L^{2}}^{2}+\|\nabla b(t)\|_{L^{2}}^{2}\right)+\left\|\nabla^{2} v(t)\right\|_{L^{2}}^{2}+\|\operatorname{div} \nabla v(t)\|_{L^{2}}^{2}+\left\|\nabla^{2} b(t)\right\|_{L^{2}}^{2} \\
= & -\int_{\mathbb{R}^{n}} \nabla(u \cdot \nabla u) \nabla u d x+\int_{\mathbb{R}^{n}} \nabla(b \cdot \nabla b) \nabla u d x-\int_{\mathbb{R}^{n}} \nabla(u \cdot \nabla v) \nabla v d x \\
& -\int_{\mathbb{R}^{n}} \nabla(u \cdot \nabla b) \nabla b d x+\int_{\mathbb{R}^{n}} \nabla(b \cdot \nabla u) \nabla b d x .
\end{aligned}
$$

By (3.3) and $\nabla \cdot u=0, \nabla \cdot b=0$, we deduce that

$$
\begin{aligned}
& \frac{1}{2} \frac{d}{d t}\left(\|\nabla u(t)\|_{L^{2}}^{2}+\|\nabla v(t)\|_{L^{2}}^{2}+\|\nabla b(t)\|_{L^{2}}^{2}\right)+\left\|\nabla^{2} v(t)\right\|_{L^{2}}^{2}+\|\operatorname{div} \nabla v(t)\|_{L^{2}}^{2}+\left\|\nabla^{2} b(t)\right\|_{L^{2}}^{2} \\
\leq & 3\|\nabla u(t)\|_{L^{\infty}}\left(\|\nabla u(t)\|_{L^{2}}^{2}+\|\nabla v(t)\|_{L^{2}}^{2}+\|\nabla b(t)\|_{L^{2}}^{2}\right) .
\end{aligned}
$$

Using Gronwall inequality, we get

$$
\begin{aligned}
& \|\nabla u(t)\|_{L^{2}}^{2}+\|\nabla v(t)\|_{L^{2}}^{2}+\|\nabla b(t)\|_{L^{2}}^{2}+2 \int_{t_{0}}^{t}\left\|\nabla^{2} v(\tau)\right\|_{L^{2}}^{2} d \tau+ \\
& 2 \int_{t_{0}}^{t}\|\operatorname{div} \nabla v(\tau)\|_{L^{2}}^{2} d \tau+2 \int_{t_{0}}^{t}\left\|\nabla^{2} b(\tau)\right\|_{L^{2}}^{2} d \tau \\
\leq & \left(\left\|\nabla u\left(t_{0}\right)\right\|_{L^{2}}^{2}+\left\|\nabla v\left(t_{0}\right)\right\|_{L^{2}}^{2}+\left\|\nabla b\left(t_{0}\right)\right\|_{L^{2}}^{2}\right) \exp \left\{C \int_{t_{0}}^{t}\|\nabla u(\tau)\|_{L^{\infty}} d \tau\right\} .
\end{aligned}
$$

Owing to (1.3), we know that for any small constant $\varepsilon>0$, there exists $T *<T$ such that

$$
\int_{T_{\star}}^{T}\|\nabla \times u(t)\|_{\dot{B}_{\infty, \infty}^{0}} d t \leq \varepsilon .
$$

Let

$$
\Theta(t)=\sup _{T_{\star} \leq \tau \leq t}\left(\left\|\nabla^{3} u(\tau)\right\|_{L^{2}}^{2}+\left\|\nabla^{3} v(\tau)\right\|_{L^{2}}^{2}+\left\|\nabla^{3} b(\tau)\right\|_{L^{2}}^{2}\right), T_{\star} \leq t<T .
$$

It follows from (3.5), (3.6), (3.7) and Lemma 2.4 that

$$
\begin{aligned}
& \|\nabla u(t)\|_{L^{2}}^{2}+\|\nabla v(t)\|_{L^{2}}^{2}+\|\nabla b(t)\|_{L^{2}}^{2}+2 \int_{T_{\star}}^{t}\left\|\nabla^{2} v(\tau)\right\|_{L^{2}}^{2} d \tau+ \\
& 2 \int_{T_{\star}}^{t}\|\operatorname{div} \nabla v(\tau)\|_{L^{2}}^{2} d \tau+2 \int_{T_{\star}}^{t}\left\|\nabla^{2} b(\tau)\right\|_{L^{2}}^{2} d \tau \\
\leq & C_{1} \exp \left\{C_{0} \int_{T_{\star}}^{t}\|\nabla \times u\|_{\dot{B}_{\infty, \infty}^{0}} \ln \left(e+\|u\|_{H^{3}}\right) d \tau\right\} \\
\leq & C_{1} \exp \left\{C_{0} \varepsilon \ln (e+\Theta(t))\right\} \\
\leq & C_{1}(e+\Theta(t))^{C_{0} \varepsilon}, \quad T_{\star} \leq t<T .
\end{aligned}
$$


where $C_{1}$ depends on $\left\|\nabla u\left(T_{\star}\right)\right\|_{L^{2}}^{2}+\left\|\nabla v\left(T_{\star}\right)\right\|_{L^{2}}^{2}+\left\|\nabla b\left(T_{\star}\right)\right\|_{L^{2}}^{2}$, while $C_{0}$ is an absolute positive constant.

Applying $\nabla^{m}$ to the first equation of (1.2), then taking $L^{2}$ inner product of the resulting equation with $\nabla^{m} u$ and using integration by parts, we have

$$
\frac{1}{2} \frac{d}{d t}\left\|\nabla^{m} u(t)\right\|_{L^{2}}^{2}=-\int_{\mathbb{R}^{n}} \nabla^{m}(u \cdot \nabla u) \nabla^{m} u d x+\int_{\mathbb{R}^{n}} \nabla^{m}(b \cdot \nabla b) \nabla^{m} u d x .
$$

Likewise, we obtain

$$
\frac{1}{2} \frac{d}{d t}\left\|\nabla^{m} v(t)\right\|_{L^{2}}^{2}+\left\|\nabla^{m} \nabla v(t)\right\|_{L^{2}}^{2}+\left\|\operatorname{div} \nabla^{m} v(t)\right\|_{L^{2}}^{2}=-\int_{\mathbb{R}^{n}} \nabla^{m}(u \cdot \nabla v) \nabla^{m} v d x .
$$

and

$$
\frac{1}{2} \frac{d}{d t}\left\|\nabla^{m} b(t)\right\|_{L^{2}}^{2}+\left\|\nabla^{m} \nabla b(t)\right\|_{L^{2}}^{2}=-\int_{\mathbb{R}^{n}} \nabla^{m}(u \cdot \nabla b) \nabla^{m} b d x+\int_{\mathbb{R}^{n}} \nabla^{m}(b \cdot \nabla u) \nabla^{m} b d x .
$$

It follows (3.9), (3.10), (3.11), $\nabla \cdot u=0, \nabla \cdot b=0$ and integration by parts that

$$
\begin{aligned}
& \frac{1}{2} \frac{d}{d t}\left(\left\|\nabla^{m} u(t)\right\|_{L^{2}}^{2}+\left\|\nabla^{m} v(t)\right\|_{L^{2}}^{2}+\left\|\nabla^{m} b(t)\right\|_{L^{2}}^{2}\right)+ \\
& \left\|\nabla^{m} \nabla v(t)\right\|_{L^{2}}^{2}+\left\|\operatorname{div} \nabla^{m} v(t)\right\|_{L^{2}}^{2}+\left\|\nabla^{m} \nabla b(t)\right\|_{L^{2}}^{2} \\
= & -\int_{\mathbb{R}^{n}}\left[\nabla^{m}(u \cdot \nabla u)-u \cdot \nabla \nabla^{m} u\right] \nabla^{m} u d x+\int_{\mathbb{R}^{n}}\left[\nabla^{m}(b \cdot \nabla b)-b \cdot \nabla \nabla^{m} b\right] \nabla^{m} u d x \\
& -\int_{\mathbb{R}^{n}}\left[\nabla^{m}(u \cdot \nabla v)-u \cdot \nabla \nabla^{m} v\right] \nabla^{m} v d x-\int_{\mathbb{R}^{n}}\left[\nabla^{m}(u \cdot \nabla b)-u \cdot \nabla \nabla^{m} b\right] \nabla^{m} b d x \\
& +\int_{\mathbb{R}^{n}}\left[\nabla^{m}(b \cdot \nabla u)-b \cdot \nabla \nabla^{m} u\right] \nabla^{m} b d x .
\end{aligned}
$$

In what follows, for simplicity, we will set $m=3$.

With help of Hölder inequality and Lemma 2.3, we derive

$$
\left|-\int_{\mathbb{R}^{n}}\left[\nabla^{3}(u \cdot \nabla u)-u \cdot \nabla \nabla^{3} u\right] \nabla^{3} u d x\right| \leq C\|\nabla u(t)\|_{L^{\infty}}\left\|\nabla^{3} u(t)\right\|_{L^{2}}^{2} .
$$

Using integration by parts and Hölder inequality, we get

$$
\begin{aligned}
& \quad\left|-\int_{\mathbb{R}^{n}}\left[\nabla^{3}(u \cdot \nabla v)-u \cdot \nabla \nabla^{3} v\right] \nabla^{3} v d x\right| \\
\leq & 7\|\nabla u(t)\|_{L^{\infty}}\left\|\nabla^{3} v(t)\right\|_{L^{2}}^{2}+4\|\nabla u(t)\|_{L^{\infty}}\left\|\nabla^{2} v(t)\right\|_{L^{2}}\left\|\nabla^{4} v(t)\right\|_{L^{2}+} \\
& \left\|\nabla^{2} u(t)\right\|_{L^{4}}\|\nabla v(t)\|_{L^{4}}\left\|\nabla^{4} v(t)\right\|_{L^{2}} .
\end{aligned}
$$

Thanks to Lemma 2.5, Young inequality and (3.8), we get

$$
\begin{aligned}
& 4\|\nabla u(t)\|_{L^{\infty}}\left\|\nabla^{2} v(t)\right\|_{L^{2}}\left\|\nabla^{4} v(t)\right\|_{L^{2}} \\
\leq & C\|\nabla u(t)\|_{L^{\infty}}\|\nabla v(t)\|_{L^{2}}^{\frac{2}{3}}\left\|\nabla^{4} v(t)\right\|_{L^{2}}^{\frac{4}{3}}
\end{aligned}
$$$$
\leq \frac{1}{4}\left\|\nabla^{4} v(t)\right\|_{L^{2}}^{2}+C\|\nabla u(t)\|_{L^{\infty}}^{3}\|\nabla v(t)\|_{L^{2}}^{2}
$$$$
\leq \frac{1}{4}\left\|\nabla^{4} v(t)\right\|_{L^{2}}^{2}+C\|\nabla u(t)\|_{L^{\infty}}\|\nabla u(t)\|_{L^{2}}^{\frac{1}{2}}\left\|\nabla^{3} u(t)\right\|_{L^{2}}^{\frac{3}{2}}\|\nabla v(t)\|_{L^{2}}^{2}
$$$$
\leq \frac{1}{4}\left\|\nabla^{4} v(t)\right\|_{L^{2}}^{2}+C\|\nabla u(t)\|_{L^{\infty}}(e+\Theta(t))^{\frac{5}{4} C_{0} \varepsilon} \Theta^{\frac{3}{4}}(t)
$$ 
in $3 \mathrm{D}$ and

$4\|\nabla u(t)\|_{L^{\infty}}\left\|\nabla^{2} v(t)\right\|_{L^{2}}\left\|\nabla^{4} v(t)\right\|_{L^{2}}$

$\leq C\|\nabla u(t)\|_{L^{\infty}}\|\nabla v(t)\|_{L^{2}}^{\frac{2}{3}}\left\|\nabla^{4} v(t)\right\|_{L^{2}}^{\frac{4}{3}}$

$\leq \frac{1}{4}\left\|\nabla^{4} v(t)\right\|_{L^{2}}^{2}+C\|\nabla u(t)\|_{L^{\infty}}^{3}\|\nabla v(t)\|_{L^{2}}^{2}$

$\leq \frac{1}{4}\left\|\nabla^{4} v(t)\right\|_{L^{2}}^{2}+C\|\nabla u(t)\|_{L^{\infty}}\|\nabla u(t)\|_{L^{2}}\left\|\nabla^{3} u(t)\right\|_{L^{2}}\|\nabla v(t)\|_{L^{2}}^{2}$

$\leq \frac{1}{4}\left\|\nabla^{4} v(t)\right\|_{L^{2}}^{2}+C\|\nabla u(t)\|_{L^{\infty}}(e+\Theta(t))^{\frac{3}{2} C_{0} \varepsilon} \Theta^{\frac{1}{2}}(t)$

in 2D.

It follows from Lemmas 2.2, 2.5, Young inequality and (3.8) that

$\left\|\nabla^{2} u(t)\right\|_{L^{4}}\|\nabla v(t)\|_{L^{4}}\left\|\nabla^{4} v(t)\right\|_{L^{2}}$

$\leq C\|\nabla u(t)\|_{L^{\infty}}^{\frac{1}{2}}\left\|\nabla^{3} u(t)\right\|_{L^{2}}^{\frac{1}{2}}\|\nabla v(t)\|_{L^{2}}^{\frac{3}{4}}\left\|\nabla^{4} v(t)\right\|_{L^{2}}^{\frac{5}{4}}$

$\leq \frac{1}{4}\left\|\nabla^{4} v(t)\right\|_{L^{2}}^{2}+C\|\nabla u(t)\|_{L^{\infty}}^{\frac{4}{3}}\left\|\nabla^{3} u(t)\right\|_{L^{2}}^{\frac{4}{3}}\|\nabla v(t)\|_{L^{2}}^{2}$

$\leq \frac{1}{4}\left\|\nabla^{4} v(t)\right\|_{L^{2}}^{2}+C\|\nabla u(t)\|_{L^{\infty}}\|\nabla u(t)\|_{L^{2}}^{\frac{1}{12}}\left\|\nabla^{3} u(t)\right\|_{L^{2}}^{\frac{19}{12}}\|\nabla v(t)\|_{L^{2}}^{2}$

$\leq \frac{1}{4}\left\|\nabla^{4} v(t)\right\|_{L^{2}}^{2}+C\|\nabla u(t)\|_{L^{\infty}}(e+\Theta(t))^{\frac{25}{24} C_{0} \varepsilon} \Theta^{\frac{19}{24}}(t)$

in $3 \mathrm{D}$ and

$$
\begin{aligned}
& \left\|\nabla^{2} u(t)\right\|_{L^{4}}\|\nabla v(t)\|_{L^{4}}\left\|\nabla^{4} v(t)\right\|_{L^{2}} \\
\leq & C\|\nabla u(t)\|_{L^{\infty}}^{\frac{1}{2}}\left\|\nabla^{3} u(t)\right\|_{L^{2}}^{\frac{1}{2}}\|\nabla v(t)\|_{L^{2}}^{\frac{5}{6}}\left\|\nabla^{4} v(t)\right\|_{L^{2}}^{\frac{7}{6}} \\
\leq & \frac{1}{4}\left\|\nabla^{4} v(t)\right\|_{L^{2}}^{2}+C\|\nabla u(t)\|_{L^{\infty}}^{\frac{6}{5}}\left\|\nabla^{3} u(t)\right\|_{L^{2}}^{\frac{6}{5}}\|\nabla v(t)\|_{L^{2}}^{2} \\
\leq & \frac{1}{4}\left\|\nabla^{4} v(t)\right\|_{L^{2}}^{2}+C\|\nabla u(t)\|_{L^{\infty}}\|\nabla u(t)\|_{L^{2}}^{\frac{1}{10}}\left\|\nabla^{3} u(t)\right\|_{L^{2}}^{\frac{13}{10}}\|\nabla v(t)\|_{L^{2}}^{2} \\
\leq & \frac{1}{4}\left\|\nabla^{4} v(t)\right\|_{L^{2}}^{2}+C\|\nabla u(t)\|_{L^{\infty}}(e+\Theta(t))^{\frac{21}{20} C_{0} \varepsilon} \Theta^{\frac{13}{20}}(t)
\end{aligned}
$$

in $2 \mathrm{D}$.

Consequently, we get

$$
\begin{gathered}
4\|\nabla u(t)\|_{L^{\infty}}\left\|\nabla^{2} v(t)\right\|_{L^{2}}\left\|\nabla^{4} v(t)\right\|_{L^{2}} \\
\leq \frac{1}{4}\left\|\nabla^{4} v(t)\right\|_{L^{2}}^{2}+C\|\nabla u(t)\|_{L^{\infty}}(e+\Theta(t))
\end{gathered}
$$

and

$$
\begin{aligned}
\left\|\nabla^{2} u(t)\right\|_{L^{4}}\|\nabla v(t)\|_{L^{4}}\left\|\nabla^{4} v(t)\right\|_{L^{2}} \\
\leq \frac{1}{4}\left\|\nabla^{4} v(t)\right\|_{L^{2}}^{2}+C\|\nabla u(t)\|_{L^{\infty}}(e+\Theta(t))
\end{aligned}
$$

provided that

$$
\varepsilon \leq \frac{1}{5 C_{0}}
$$


It follows from (3.14), (3.15) and (3.16) that

$$
\begin{gathered}
\quad\left|-\int_{\mathbb{R}^{n}}\left[\nabla^{3}(u \cdot \nabla v)-u \cdot \nabla \nabla^{3} v\right] \nabla^{3} v d x\right| \\
\leq \frac{1}{2}\left\|\nabla^{4} v(t)\right\|_{L^{2}}^{2}+C\|\nabla u(t)\|_{L^{\infty}}(e+\Theta(t)) .
\end{gathered}
$$

Likewise, we have

$$
\begin{aligned}
& \left|-\int_{\mathbb{R}^{n}}\left[\nabla^{3}(u \cdot \nabla b)-u \cdot \nabla \nabla^{3} b\right] \nabla^{3} b d x\right| \\
\leq & \frac{1}{6}\left\|\nabla^{4} b(t)\right\|_{L^{2}}^{2}+C\|\nabla u(t)\|_{L^{\infty}}(e+\Theta(t)) . \\
& \left|\int_{\mathbb{R}^{n}}\left[\nabla^{3}(b \cdot \nabla b)-b \cdot \nabla \nabla^{3} b\right] \nabla^{3} u d x\right| \\
\leq & \frac{1}{6}\left\|\nabla^{4} b(t)\right\|_{L^{2}}^{2}+C\|\nabla u(t)\|_{L^{\infty}}(e+\Theta(t))
\end{aligned}
$$

and

$$
\begin{aligned}
& \left|\int_{\mathbb{R}^{n}}\left[\nabla^{3}(b \cdot \nabla u)-b \cdot \nabla \nabla^{3} u\right] \nabla^{3} b d x\right| \\
\leq & \frac{1}{6}\left\|\nabla^{4} b(t)\right\|_{L^{2}}^{2}+C\|\nabla u(t)\|_{L^{\infty}}(e+\Theta(t))
\end{aligned}
$$

Collecting (3.12), (3.13), (3.17), (3.18), (3.19) and (3.20) yields

$$
\begin{aligned}
& \frac{d}{d t}\left(\left\|\nabla^{3} u(t)\right\|_{L^{2}}^{2}+\left\|\nabla^{3} v(t)\right\|_{L^{2}}^{2}+\left\|\nabla^{3} b(t)\right\|_{L^{2}}^{2}\right)+\left\|\nabla^{4} v(t)\right\|_{L^{2}}^{2}+ \\
& \left\|\operatorname{div} \nabla^{3} v(t)\right\|_{L^{2}}^{2}+\left\|\nabla^{4} b(t)\right\|_{L^{2}}^{2} \\
\leq & C\|\nabla u(t)\|_{L^{\infty}}(e+\Theta(t))
\end{aligned}
$$

for all $T^{*} \leq t<T$.

Integrating (3.21) with respect to time from $T$ " to $\tau$ and using Lemma 2.4, we have

$$
\begin{aligned}
& e+\left\|\nabla^{3} u(\tau)\right\|_{L^{2}}^{2}+\left\|\nabla^{3} v(\tau)\right\|_{L^{2}}^{2}+\left\|\nabla^{3} b(\tau)\right\|_{L^{2}}^{2} \\
\leq & e+\left\|\nabla^{3} u\left(T_{\star}\right)\right\|_{L^{2}}^{2}+\left\|\nabla^{3} v\left(T_{\star}\right)\right\|_{L^{2}}^{2}+\left\|\nabla^{3} b\left(T_{\star}\right)\right\|_{L^{2}}^{2}+ \\
& C_{2} \int_{T_{\star}}^{\tau}\left[1+\|u\|_{L^{2}}+\|\nabla \times u(s)\|_{\dot{B}_{\infty, \infty}^{0}} \ln (e+\Theta(s))\right](e+\Theta(s)) d s .
\end{aligned}
$$

Owing to (3.22), we get

$$
\begin{aligned}
e+A(t) \leq & e+\left\|\nabla^{3} u\left(T_{\star}\right)\right\|_{L^{2}}^{2}+\left\|\nabla^{3} v\left(T_{\star}\right)\right\|_{L^{2}}^{2}+\left\|\nabla^{3} b\left(T_{\star}\right)\right\|_{L^{2}}^{2}+ \\
& C_{2} \int_{T_{\star}}^{t}\left[1+\|u\|_{L^{2}}+\|\nabla \times u(\tau)\|_{\dot{B}_{\infty, \infty}^{0}} \ln (e+\Theta(\tau))\right](e+\Theta(\tau)) d \tau .
\end{aligned}
$$

For all $T^{*} \leq t<T$, with help of Gronwall inequality and (3.23), we have

$$
e+\left\|\nabla^{3} u(t)\right\|_{L^{2}}^{2}+\left\|\nabla^{3} v(t)\right\|_{L^{2}}^{2}+\left\|\nabla^{3} b(t)\right\|_{L^{2}}^{2} \leq C,
$$

where $C$ depends on $\left\|\nabla u\left(T_{\star}\right)\right\|_{L^{2}}^{2}+\left\|\nabla v\left(T_{\star}\right)\right\|_{L^{2}}^{2}+\left\|\nabla b\left(T_{\star}\right)\right\|_{L^{2}}^{2}$.

Noting that (3.2) and the right-hand side of (3.24) is independent of $t$ for $T_{0} \leq t<T$, we know that $(u(T, \cdot), v(T, \cdot), b(T, \cdot)) \in H^{3}\left(\mathbb{R}^{n}\right)$. Thus, Theorem 1.1 is proved. 


\section{Acknowledgements}

The authors would like to thank the referee for his/her pertinent comments and advice. This work was supported in part by Research Initiation Project for High-level Talents (201031) of North China University of Water Resources and Electric Power.

\section{Authors' contributions}

YZW completed the main part of theorem in this paper, YL and YXW revised the part proof. All authors read and approve the final manuscript.

\section{Competing interests}

The authors declare that they have no competing interests.

Received: 4 April 2011 Accepted: 15 August 2011 Published: 15 August 2011

\section{References}

1. Gala, S: Regularity criteria for the 3D magneto-micropolar fluid equations in the Morrey-Campanato space. Nonlinear Differ Equ Appl. 17, 181-194 (2010). doi:10.1007/s00030-009-0047-4

2. Wang, Y, Hu, L, Wang, Y: A Beale-Kato Majda criterion for magneto-micropolar fluid equations with partial viscosity. Bound Value Prob 2011, 14 (2011). Article ID 128614. doi:10.1186/1687-2770-2011-14

3. Ortega-Torres, E, Rojas-Medar, M: On the uniqueness and regularity of the weak solution for magneto-micropolar fluid equations. Revista de Matemáticas Aplicadas. 17, 75-90 (1996)

4. Ortega-Torres, E, Rojas-Medar, M: Magneto-micropolar fluid motion: global existence of strong solutions. Abstract Appl Anal. 4, 109-125 (1999). doi:10.1155/S1085337599000287

5. Rojas-Medar, M: Magneto-micropolar fluid motion: existence and uniqueness of strong solutions. Mathematische Nachrichten. 188, 301-319 (1997). doi:10.1002/mana.19971880116

6. Rojas-Medar, M, Boldrini, J: Magneto-micropolar fluid motion: existence of weak solutions. Rev Mat Complut. 11, 443-460 (1998)

7. Yuan, B: regularity of weak solutions to magneto-micropolar fluid equations. Acta Mathematica Scientia. 30, 1469-1480 (2010). doi:10.1016/50252-9602(10)60139-7

8. Yuan, J: Existence theorem and blow-up criterion of the strong solutions to the magneto-micropolar fluid equations. Math Methods Appl Sci. 31, 1113-1130 (2008). doi:10.1002/mma.967

9. Eringen, A: Theory of micropolar fluids. J Math Mech. 16, 1-18 (1966)

10. Lukaszewicz, G: Micropolar fluids. Theory and Applications, Modeling and Simulation in Science, Engineering and Technology. Birkhäuser, Baston. (1999)

11. Galdi, G, Rionero, S: A note on the existence and uniqueness of solutions of the micropolar fluid equations. Int J Eng Sci. 15, 105-108 (1977). doi:10.1016/0020-7225(77)90025-8

12. Yamaguchi, N: Existence of global strong solution to the micropolar fluid system in a bounded domain. Math Methods Appl Sci. 28, 1507-1526 (2005). doi:10.1002/mma.617

13. Yuan, B: On regularity criteria for weak solutions to the micropolar fluid equations in Lorentz space. Proc Am Math Soc 138, 2025-2036 (2010). doi:10.1090/50002-9939-10-10232-9

14. Fan, J, Zhou, Y, Zhu, M: A regularity criterion for the 3D micropolar fluid flows with zero angular viscosity. (2010, in press)

15. Fan, J, He, X: A regularity criterion of the 3D micropolar fluid flows. (2011, in press)

16. Fan, J, Jin, L: A regularity criterion of the micropolar fluid flows. (2011, in press)

17. Dong, B, Chen, Z: Regularity criteria of weak solutions to the three-dimensional micropolar flows. J Math Phys. 50 103525-1-103525-13 (2009)

18. Szopa, P: Gevrey class regularity for solutions of micropolar fluid equations. J Math Anal Appl. 351, 340-349 (2009). doi:10.1016/j.jmaa.2008.10.026

19. Ortega-Torres, E, Rojas-Medar, M: On the regularity for solutions of the micropolar fluid equations. Rendiconti del Seminario Matematico della Università de Padova. 122, 27-37 (2009)

20. Ortega-Torres, E, Rojas-Medar, M, Villamizar-Roa, EJ: Micropolar fluids with vanishing viscosity. Abstract Appl Anal 2010 18 (2010). Article ID 843692

21. Sermange, $M$, Temam, R: Some mathematical questions related to the MHD equations. Commun Pure Appl Math. 36, 635-666 (1983). doi:10.1002/cpa.3160360506

22. Caisch, R, Klapper, I, Steele, G: Remarks on singularities, dimension and energy dissipation for ideal hydrodynamics and MHD. Commun Math Phys. 184, 443-455 (1997). doi:10.1007/s002200050067

23. Cannone, $\mathrm{M}$, Chen, $\mathrm{Q}$, Miao, C: A losing estimate for the ideal MHD equations with application to blow-up criterion. SIAM J Math Anal. 38, 1847-1859 (2007). doi:10.1137/060652002

24. Cao, C, Wu, J: Two regularity criteria for the 3D MHD equations. J Diff Equ. 248, 2263-2274 (2010). doi:10.1016/j. jide.2009.09.020

25. He, C, Xin, Z: Partial regularity of suitable weak sokutions to the incompressible magnetohydrodynamics equations. J Funct Anal. 227, 113-152 (2005). doi:10.1016/.j.ja.2005.06.009

26. Lei, Z, Zhou, Y: BKM criterion and global weak solutions for Magnetohydrodynamics with zero viscosity. Discrete Contin Dyn Syst A. 25, 575-583 (2009)

27. Wu, J: Regularity results for weak solutions of the 3D MHD equations. Discrete Contin Dyn Syst. 10, 543-556 (2004)

28. Wu, J: Regularity criteria for the generalized MHD equations. Commun Partial Differ Equ. 33, 285-306 (2008). doi:10.1080/03605300701382530

29. Zhou, Y: Remarks on regularities for the 3D MHD equations. Discrete Contin Dyn Syst. 12, 881-886 (2005)

30. Zhou, Y: Regularity criteria for the 3D MHD equations in term of the pressure. Int J Nonlinear Mech. 41,1174-1180 (2006). doi:10.1016/j.jnonlinmec.2006.12.001 
31. Zhou, Y: Regularity criteria for the generalized viscous MHD equations. Ann Inst H Poincaré Anal Non Linéaire. 24 491-505 (2007). doi:10.1016/j.anihpc.2006.03.014

32. Zhou, Y, Gala, S: Regularity criteria for the solutions to the 3D MHD equations in the multiplier space. Z Angew Math Phys. 61, 193-199 (2010). doi:10.1007/s00033-009-0023-1

33. Zhou, Y, Gala, S: A new regularity criterion for weak solutions to the viscous MHD equations in terms of the vorticity field. Nonlinear Anal. 72, 3643-3648 (2010). doi:10.1016/j.na.2009.12.045

34. Zhou, Y, Fan, J: Logarithmically improved regularity criteria for the 3D viscous MHD equations. Forum Math. (2010, in press)

35. Beale, J, Kato, T, Majda, A: Remarks on the breakdown of smooth solutions for the 3D Euler equations. Commun Math Phys. 94, 61-66 (1984). doi:10.1007/BF01212349

36. Kozono, H, Ogawa, T, Taniuchi, Y: The critical Sobolev inequalities in Besov spaces and regularity criterion to some semi-linear evolution equations. Math Z. 242, 251-278 (2002). doi:10.1007/s002090100332

37. Triebel, H: Theory of Function Spaces. Monograph in Mathematics. Birkhauser, Basel. 78 (1983)

38. Chemin, J: Perfect Incompressible Fluids. In Oxford Lecture Ser Math Appl, vol. 14,The Clarendon Press/Oxford University Press, New York (1998)

39. Majda, A, Bertozzi, A: Vorticity and Incompressible Flow. Cambridge University Press, Cambridge (2002)

doi:10.1186/1687-2770-2011-11

Cite this article as: Wang et al:: Blow-up criterion of smooth solutions for magneto-micropolar fluid equations with partial viscosity. Boundary Value Problems 2011 2011:11.

\section{Submit your manuscript to a SpringerOpen ${ }^{\odot}$} journal and benefit from:

- Convenient online submission

- Rigorous peer review

- Immediate publication on acceptance

- Open access: articles freely available online

- High visibility within the field

- Retaining the copyright to your article

Submit your next manuscript at $\gg$ springeropen.com 